\title{
EFEITO DOS PARÂMETROS DO MODELO DE HERTZ-MINDLIN DE FORÇAS DE CONTATO ENTRE PARTÍCULAS SOBRE O ÂNGULO DE REPOUSO ESTÁTICO DA SOJA
}

\author{
R. C. SANTANA ${ }^{1}$, K. G. SANTOS ${ }^{2}$, A. V. P. CAMPOS ${ }^{1}$, L. V. FERREIRA ${ }^{1}$, O. S. OLIVEIRA ${ }^{1}$ e \\ M. A. S. BARROZO ${ }^{1}$ \\ ${ }^{1}$ Universidade Federal de Uberlândia, Faculdade de Engenharia Química \\ ${ }^{2}$ Universidade Federal Do Triângulo Mineiro, Departamento de Engenharia Química \\ *e-mail: kassiagsantos@gmail.com
}

\begin{abstract}
RESUMO - O ângulo de repouso estático é uma característica própria do material particulado e é uma função da densidade da partícula, área superficial e forma, além do coeficiente de atrito do material. Neste trabalho, o ângulo de repouso estático de sementes de soja foram obtidos experimentalmente e por simulação simulados empregando o Método de Elementos Discretos (DEM). As forças de contato entre as partículas foram representadas pelo Modelo Hertz-Mindlin. A fim de verificar como os parâmetros do modelo de força de contato influenciavam o valor do ângulo de repouso, a técnica de planejamento composto central foi empregada para delinear as simulações. Os resultados mostraram que quais os parâmetros que mais influenciam o comportamento granular da soja. Também foi possível encontrar um conjunto de valores para os parâmetros que produziram um resultado simulado muito próximo ao valor experimental.
\end{abstract}

\section{INTRODUÇÃO}

Vários dos processos industriais envolvem operações com partículas. A descrição matemática do escoamento de sistemas multifásicos granulares é atualmente uma ferramenta com grande potencial para auxiliar na compreensão do processo de transporte de sólidos. Essa modelagem é complexa e pode ser efetuada por diferentes abordagens (como a Lagrangeana e a Euleriana), que apresentam vantagens e limitações (Santos et al., 2009).

No caso de leitos móveis, a modelagem utilizando a aproximação Euler-Euler, que trata as fases como fluidas e interpenetrantes, apresenta boa predição para sistemas particulados densos, principalmente com partículas esféricas e de maior densidade. De acordo com Gao e Sun (), a maior falha da abordagem Euler-Euler aplicada a sistemas particulados é que ele não captura as características individuais das partículas sólidas quanto à sua forma e tamanho e não pode identificar efetivamente a influência dessas características na fluidodinâmica da partícula. Isso ocorre principalmente para sistemas com materiais particulados heterogêneos, com baixa esfericidade e baixa densidade (implica em choques menos elásticos), como é o caso das biomassas em geral.

Segundo Souza (2010), um dos maiores desafios da modelagem de sistemas granulares é ainda 


\section{9 a 22 de outubro de 2014 \\ Florianópolis/SC}

modelar todas as características desse complexo escoamento, principalmente em se tratando de sistemas densos. Para uma simulação a nível microscópico, cada partícula deve ser considerada como um sistema que tem seu movimento descrito pelas Leis de Newton. Nesse aspecto, novas metodologias computacionais têm sido desenvolvidas, como o Método de Elementos Discretos (DEM).

O Método de Elementos Discretos, tem se mostrado uma boa alternativa para a modelagem de sistemas particulados densos, pois contabiliza o efeito do tamanho e da forma real da partícula, além de permitir uma modelagem detalhada da colisão entre as partículas com outras partículas e com a parede do recipiente.

Nota-se que os valores encontrados para os parâmetros dos modelos nos trabalhos de simulação DEM, variam significativamente, pois cada estudo foi concebido para equipamentos distintos e representa o escoamento de materiais diferentes os quais podem conduzir diferentes respostas de colisões partícula-partícula e partícula-parede. Sendo assim, a abordagem DEM necessita de uma calibração prévia dos parâmetros do modelo de contato entre partículas, a fim de que o choque destas na simulação seja similar ao que ocorre experimentalmente. Para tal, empregam-se testes experimentais simples, como por exemplo os ângulos de repouso estático e dinâmico das partículas.

O ângulo de repouso estático é definido como o ângulo interno entre a superfície lateral da pilha e o plano horizontal e é uma característica própria do material particulado, que é função da densidade da partícula, área superficial e forma, além do coeficiente de atrito do material. Walton e Braun (1993) investigaram o efeito da forma da partícula no ângulo de repouso dinâmico e estático usando DEM, usando esferas e cubos. Eles concluíram que o ângulos de repouso estático e dinâmico aumentam com o aumento da não-esfericidade da partícula.

Neste trabalho o ângulo de repouso estático de sementes de soja foram obtidos experimentalmente e simulados pelo Método de elementos discretos (DEM) empregando o software EDEM 2.3. As forças de contato entre as partículas foram representadas pelo Modelo Hertz-Mindlin. A fim de verificar como os parâmetros do modelo de força de contato influenciavam o valor do ângulo de repouso, a técnica de planejamento composto central foi empregada para delinear as simulações.

\section{O MÉTODO DE ELEMENTOS DISCRETOS}

O Método de Elementos Discretos (DEM) envolve a descrição do movimento de cada partícula no escoamento e a modelagem da colisão entre as partículas e os contornos rígidos, sendo o método mais adequado para lidar com problemas de natureza descontínua, e ao mesmo tempo com grandes níveis de deformação, como é o caso do escoamento granular.

A metodologia DEM e suas variantes estão bem estabelecidas e são descritas em detalhes por Cleary (2008). O modelo de contato, usado para contabilizar as forças de interação entre duas esferas rígidas que pertencem a duas diferentes partículas, é baseado no modelo de Hertz-Mindlin, que 
caracteriza as interações por meio de três coeficientes: coeficiente de restituição, coeficiente de atrito estático e coeficiente de atrito de rolamento.

No modelo de colisão de Hertz-Mindlin, a componente normal da força é baseada na teoria de contato de Hertz (1882), enquanto a força tangencial é baseada no trabalho de Mindlin (1949). Tanto no cálculo da força normal, quanto da tangencial, há um termo de amortecimento do sistema, relacionado ao coeficiente de restituição (Tsuji et al., 1992), que relaciona a velocidade de separação e a velocidade de aproximação de uma ou mais partículas em uma colisão.

A força normal $\left(F_{n}\right)$ é uma função da sobreposição na direção normal ( $\left.\delta_{n} k\right)$ dada por:

$$
F_{n}=\frac{4}{3} E^{*} \sqrt{R^{*}} \delta_{n}^{3 / 2}
$$

no qual o módulo Equivalente de Young $\left(E^{*}\right)$ e o raio equivalente $\left(R^{*}\right)$ são definidos como:

$$
\begin{aligned}
\frac{1}{E^{*}} & =\frac{\left(1-v_{i}^{2}\right)}{E_{i}}+\frac{\left(1-v_{j}^{2}\right)}{E_{j}} \\
\frac{1}{R^{*}} & =\frac{1}{R_{i}}+\frac{1}{R_{j}}
\end{aligned}
$$

onde $E_{i}, v_{i}, R_{j}$ e $E_{j}, v_{j}, R_{j}$ são o modulo de Young, razão de Poisson e o raio de cada partícula em contato. Adicionalmente, há a força normal de amortecimento $\left(F_{n}^{d}\right)$ dada por:

$$
F_{n}^{d}=-2 \sqrt{\frac{5}{6}}\left[\frac{\ln e}{\sqrt{\ln ^{2} e+\pi^{2}}}\right] \sqrt{\left(2 E^{*} \sqrt{R^{*} \delta_{n}}\right) m^{*}} v_{n}^{\text {rel }}
$$

onde $m^{*}=\left(m_{1}^{-1}+m_{i}^{-1}\right)^{-1}$ é a massa equivalente, $v_{n}^{\overline{r e l}}$ é velocidade relativa na direção normal e $e$ é o coeficiente de restituição. A força tangencial $F_{t}$ depende da sobreposição na direção tangencial $\left(\delta_{t}\right) \mathrm{e}$ constante de rigidez $\left(S_{t}\right)$ :

$$
F_{t}=-\delta_{t} S_{t}=-\delta_{t} 8 G^{*} \sqrt{R^{*} \delta_{n}}
$$

na qual $G^{*}$ é o módulo cisalhante. A força de amortecimento tangencial, conhecida também como força de repulsão, é dada por:

$$
F_{t}^{d}=-2 \sqrt{\frac{5}{6}}\left[\frac{\ln e}{\sqrt{\ln ^{2} e+\pi^{2}}}\right] \sqrt{S_{t} m^{*}} v_{t}^{\overrightarrow{r e l}}
$$

sendo $v_{t}^{\overline{r e l}}$ a velocidade relativa tangencial. 
A força tangencial é limitada pelo atrito estático definido por Coulomb $\left(\mu_{s} F_{n}\right)$, onde $\mu_{s}$ é o coeficiente de atrito estático. O coeficiente de atrito de rolamento modela o efeito da rugosidade da superfície de partículas não esféricas. De fato, como a partícula irregular é representada por um conjunto de esferas (método multiesfera), elas não podem rolar umas sobre as outras sem a definição deste coeficiente. Para tal, o coeficiente de atrito de rolamento introduz um torque artificial que no modelo de contato que se opõe ao movimento de rolamento, como segue:

$$
\tau_{i}=-\mu_{r} F_{n} R_{i} \omega_{i} v
$$

no qual $\mu_{r}$ é o coeficiente de atrito de rolamento, $R_{i}$ é a distância entre o ponto de contato e o centro de massa da partícula e $\omega_{i}$ é a vetor unitário da velocidade angular no ponto de contato. O modelo da força de contato requer a informação de ambos os coeficientes de atrito das partículas e de restituição. Estes parâmetros são de difícil obtenção experimental e dependem do tipo de partícula. O algoritmo do DEM é bastante simples e pode ser descrito conforme mostra ao esquema da Figura 1.

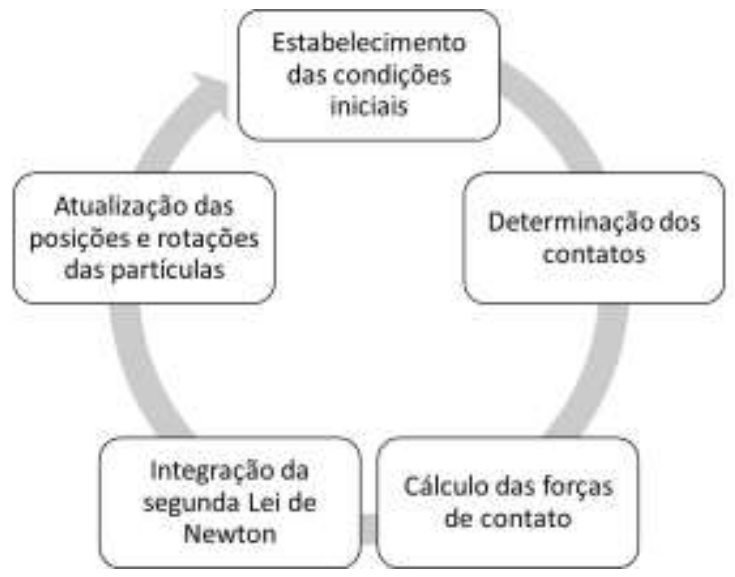

Figura 1 - Etapas básicas do ciclo de cálculo do Método de Elementos Discretos.

\section{METODOLOGIA}

\subsection{Procedimento Experimental}

O ângulo de repouso estático é definido como o ângulo interno entre a superfície de uma pilha de partículas e a superfície horizontal e está diretamente relacionado à densidade, área superficial e formato da partícula, bem como ao coeficiente de atrito do material.

Neste trabalho, o ângulo de repouso estático foi medido usando o método do funil fixo, a partir do raio e da altura do cone formado pelas partículas. O valor experimental do ângulo de repouso estático da soja foi obtido pela média de seis medidas. As sementes de soja tinham densidade aparente de $1188 \pm 6,2 \mathrm{~kg} / \mathrm{m}^{3}$ e diâmetro médio de Sauter de 6,83 $\pm 0,3 \mathrm{~mm}$, com esfericidade de 0,9. 


\subsection{Procedimento de Simulação}

O ângulo de repouso estático foi reproduzido por meio de simulações computacionais empregando DEM, implementado no software EDEM 2.3. O passo de tempo em todas as simulações foi fixado como sendo $20 \%$ do passo de tempo crítico calculado pelo tempo de Rayleigh.

O comportamento de materiais granulares é fortemente dependente do tamanho e da forma da partícula, requerendo uma representação realística da partícula. Nas simulações, realizadas neste estudo, o formato da semente de soja foi aproximado pelo método multiesfera (Favier et al., 1999), pelo agrupamento de três esferas simples, contabilizando uma partícula com volume médio de 1,69 $\cdot 10^{-7} \mathrm{~m}^{3}$, como mostra a Figura 2. Foram utilizadas nas simulações um total de 1552 partículas, que correspondem à mesma massa de partículas do experimento.

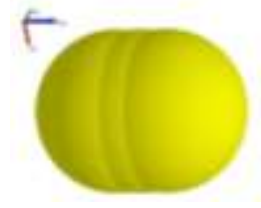

Figura 2 - Formato da semente de soja adotado nas simulações DEM.

A fim de orientar as simulações e obter um modelo matemático capaz de indicar o modo como a variação dos parâmetros de simulação influenciam no ângulo de repouso estático das partículas em estudo, foi proposto um planejamento composto central para os cinco parâmetros investigados: módulo de cisalhamento, razão de Poisson, coeficiente de restituição, coeficiente de atrito estática, coeficiente de atrito de rolamento. A Tabela 1 mostra os níveis de cada variável empregados neste estudo e sua correspondência ao valor original da variável.

Tabela 1 - Níveis e faixa de valores das variáveis codificadas

\begin{tabular}{llccccc} 
& & \multicolumn{4}{c}{ Níveis } \\
\cline { 3 - 7 } & \multicolumn{1}{c}{ Variáveis } & $-1,607$ & $-1,000$ & 0,000 & $+1,000$ & $+1,607$ \\
\hline X1 & Módulo Cisalhante [Pa] & $1,17810^{6}$ & $3,00010^{6}$ & $6,00010^{6}$ & $9,00010^{6}$ & $1,08210^{7}$ \\
X2 & Razão de Poisson & 0,018 & 0,200 & 0,500 & 0,800 & 0,982 \\
X3 & Coeficiente de Restituição & 0,018 & 0,200 & 0,500 & 0,800 & 0,982 \\
X4 & Coeficiente de Atrito Estático & 0,018 & 0,200 & 0,500 & 0,800 & 0,982 \\
X5 & Coeficiente de Atrito de Rolamento & 0,018 & 0,200 & 0,500 & 0,800 & 0,982 \\
\hline
\end{tabular}

\section{RESULTADOS}

Neste trabalho, os efeitos dos parâmetros do Modelo Hertz-Mindlin sobre o comportamento do ângulo de repouso estático da soja foram investigados por meio da técnica de superfície de resposta, a partir de um PCC, disposto na Tabela 2, na qual também encontram-se os resultados de ângulo de repouso estático obtidos nas simulações DEM $\left(\Phi_{D E M}\right)$ e o desvio $\bar{\sigma}(\mathrm{em} \%)$ entre o valor experimental e o simulado. 
Tabela 2 - O PCC empregado nas simulações DEM do ângulo de repouso estático da soja.

\begin{tabular}{|c|c|c|c|c|c|c|c|}
\hline SIMULAÇÃO & $\begin{array}{c}\text { Módulo } \\
\text { Cisalhante } \\
{[\mathrm{Pa}]} \\
\end{array}$ & $\begin{array}{c}\text { Razão de } \\
\text { Poisson }\end{array}$ & $\begin{array}{l}\text { Coeficiente } \\
\text { Restituição }\end{array}$ & $\begin{array}{c}\text { Coeficiente } \\
\text { de Atrito } \\
\text { Estático }\end{array}$ & $\begin{array}{l}\text { Coeficiente } \\
\text { de Atrito de } \\
\text { Rolamento }\end{array}$ & $\Phi_{D E M}$ & $\begin{array}{l}\bar{\sigma} . . \\
{[\%]}\end{array}$ \\
\hline 1 & $-1,000$ & $-1,000$ & $-1,000$ & $-1,000$ & 1,000 & $13,464 \pm 0,152$ & 1,132 \\
\hline 2 & $-1,000$ & $-1,000$ & $-1,000$ & 1,000 & $-1,000$ & $19,937 \pm 1,205$ & 6,042 \\
\hline 3 & $-1,000$ & $-1,000$ & 1,000 & $-1,000$ & $-1,000$ & $12,991 \pm 0,180$ & 1,389 \\
\hline 4 & $-1,000$ & $-1,000$ & 1,000 & 1,000 & 1,000 & $23,117 \pm 1,075$ & 4,650 \\
\hline 5 & $-1,000$ & 1,000 & $-1,000$ & $-1,000$ & $-1,000$ & $13,731 \pm 1,348$ & 9,814 \\
\hline 6 & $-1,000$ & 1,000 & $-1,000$ & 1,000 & 1,000 & $33,602 \pm 0,286$ & 0,851 \\
\hline 7 & $-1,000$ & 1,000 & 1,000 & $-1,000$ & 1,000 & $17,886 \pm 0,124$ & 0,693 \\
\hline 8 & $-1,000$ & 1,000 & 1,000 & 1,000 & $-1,000$ & $21,474 \pm 0,395$ & 1,842 \\
\hline 9 & 1,000 & $-1,000$ & $-1,000$ & $-1,000$ & $-1,000$ & $14,916 \pm 0,037$ & 0,250 \\
\hline 10 & 1,000 & $-1,000$ & $-1,000$ & 1,000 & 1,000 & $32,073 \pm 1,367$ & 4,262 \\
\hline 11 & 1,000 & $-1,000$ & 1,000 & $-1,000$ & 1,000 & $18,232 \pm 0,343$ & 1,883 \\
\hline 12 & 1,000 & $-1,000$ & 1,000 & 1,000 & $-1,000$ & $23,198 \pm 1,024$ & 4,414 \\
\hline 13 & 1,000 & 1,000 & $-1,000$ & $-1,000$ & 1,000 & $18,620 \pm 0,225$ & 1,207 \\
\hline 14 & 1,000 & 1,000 & $-1,000$ & 1,000 & $-1,000$ & $32,287 \pm 0,095$ & 0,295 \\
\hline 15 & 1,000 & 1,000 & 1,000 & $-1,000$ & $-1,000$ & $14,946 \pm 0,432$ & 2,892 \\
\hline 16 & 1,000 & 1,000 & 1,000 & 1,000 & 1,000 & $31,599 \pm 0,735$ & 2,326 \\
\hline 17 & $-1,607$ & 0,000 & 0,000 & 0,000 & 0,000 & $28,333 \pm 0,840$ & 2,965 \\
\hline 18 & 1,607 & 0,000 & 0,000 & 0,000 & 0,000 & $29,246 \pm 0,706$ & 2,413 \\
\hline 19 & 0,000 & $-1,607$ & 0,000 & 0,000 & 0,000 & $30,281 \pm 0,624$ & 2,061 \\
\hline 20 & 0,000 & 1,607 & 0,000 & 0,000 & 0,000 & $26,779 \pm 0,775$ & 2,896 \\
\hline 21 & 0,000 & 0,000 & $-1,607$ & 0,000 & 0,000 & $26,067 \pm 0,388$ & 1,490 \\
\hline 22 & 0,000 & 0,000 & 1,607 & 0,000 & 0,000 & $26,730 \pm 2,036$ & 7,616 \\
\hline 23 & 0,000 & 0,000 & 0,000 & $-1,607$ & 0,000 & $0,000 \pm 0,000$ & 0,000 \\
\hline 24 & 0,000 & 0,000 & 0,000 & 1,607 & 0,000 & $26,867 \pm 1,430$ & 5,321 \\
\hline 25 & 0,000 & 0,000 & 0,000 & 0,000 & $-1,607$ & $17,162 \pm 0,944$ & 5,499 \\
\hline 26 & 0,000 & 0,000 & 0,000 & 0,000 & 1,607 & $34,589 \pm 0,147$ & 0,425 \\
\hline 27 & 0,000 & 0,000 & 0,000 & 0,000 & 0,000 & $29,423 \pm 0,005$ & 0,017 \\
\hline 28 & 0,000 & 0,000 & 0,000 & 0,000 & 0,000 & $30,003 \pm 0,083$ & 0,276 \\
\hline
\end{tabular}

A Figura 3 apresenta algumas das simulações DEM. A partir dos dados da Tabela 2 e Figura 3, pode-se observar que o ângulo de repouso estático simulado varia significativamente de uma simulação pra outra, compreendendo valores entre $0^{\circ}$ e $34,6^{\circ}$. A Simulação 23 foi a única em que não se formou uma pilha com o material, mostrando que valores de coeficiente de atrito estático próximos de zero não permitem o atrito entre as partículas, fazendo com que elas deslizem umas sobre as outras com velocidade acima da real. Em contrapartida, o nível mais elevado do coeficiente de atrito de rolamento (Simulação 26) levou ao maior ângulo de repouso simulado. Já a Figura 4, compara as imagens do ângulo de repouso estático da soja obtidas experimentalmente e pela melhor condição de simulação (Simulação 8). 


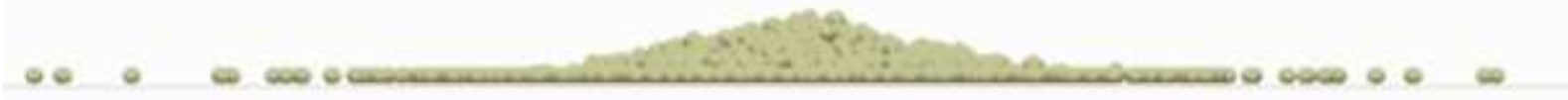

(a) Simulação $1\left(13,5^{\circ}\right)$

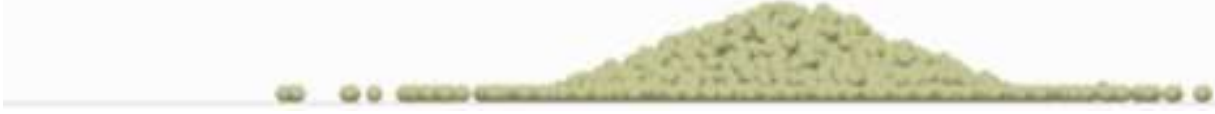

(b) Simulação $2\left(19,9^{\circ}\right)$

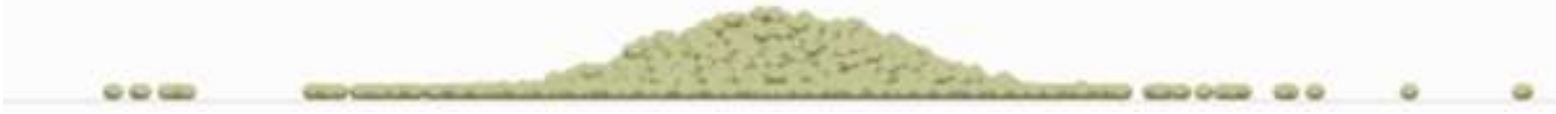

(c) Simulação $8\left(21,5^{\circ}\right)$

(d) Simulação $23\left(0,0^{\circ}\right)$

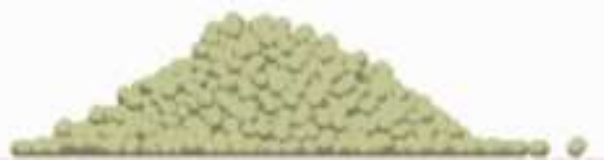

(e) Simulação $26\left(34,6^{\circ}\right)$

Figura 3 - Ângulos de repouso obtidos a partir das simulações DEM propostas no PCC.

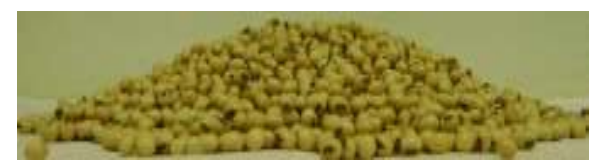

(a)

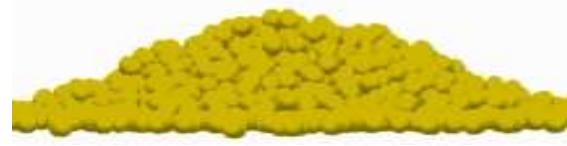

(b)

Figura 4 - Ângulo de repouso estático da soja: (a) experimental $\left(21,4^{\circ}\right)$; (b) simulado $\left(21,5^{\circ}\right)$.

Os resultados das simulações na Tabela 2 serviram como base para uma análise estatística global. Uma análise de regressão múltipla foi realizada para quantificar o efeito dos parâmetros do modelo de forças de contato de Hertz-Mindlin sobre o ângulo de repouso estático da soja. A fim de obter a equação que descreve a resposta em função das varáveis independentes, um teste de hipóteses usando a estatística t de Student para identificar os parâmetros significativos. Os coeficientes da equação estimados pelo método de mínimos quadrados com nível de significância maior que $10 \%$, $(\mathrm{p}>0,1)$ foram negligenciados. A equação de ajuste para o ângulo de repouso estático simulado $\left(\Phi_{D E M}\right)$, em notação matricial, é dada por $\left(\mathrm{R}^{2}=0,882\right)$ :

$$
\Phi_{D E M}=27,547+1,471 X_{1}+6,410 X_{4}+2,982 X_{5}-5,848 X_{4}^{2}-1,364 X_{3} X_{4}
$$

A Equação 8 mostra que a razão de Poisson não influencia significativamente a resposta e que, apesar de não ser importante de forma isolada, o coeficiente de restituição é significativo por meio da interação com o coeficiente de atrito estático. Os demais parâmetros influenciam o valor do ângulo de forma diretamente proporcional, sendo que apenas o coeficiente de atrito estático foi significativo na forma quadrática. Os parâmetros mais significativos foram os coeficientes de atrito estático seguido pelo coeficiente de atrito de rolamento. 


\section{9 a 22 de outubro de 2014 \\ Florianópolis/SC}

Como é grande a quantidade de parâmetros do modelo de força de contato, ao invés de uma única condição similar ao experimento, existe uma região com combinações de parâmetros que levam à respostas próximas da experimental. Já que o comportamento fluidodinâmico das partículas é fortemente influenciado pelos coeficientes de atrito estático e de rolamento, seria adequado que estes parâmetros fossem também medidos de forma experimental e seus valores comparados com os valores utilizados nas simulações que tiveram maior concordância com os dados experimentais. A calibração de parâmetros é uma forma simples de obter os parâmetros que representam o comportamento fluidodinâmico real de partículas em sistemas com empacotamento denso.

\section{CONCLUSÕES}

Neste trabalho, propôs-se encontrar os parâmetros do Modelo Hertz-Mindlin que melhor representam o comportamento fluidodinâmico da soja. O ângulo de repouso estático foi selecionado para a calibração paramétrica. Um PCC foi proposto para investigar os resultados de uma larga faixa dos parâmetros. A análise de regressão múltipla dos ângulos obtidos nas simulações revelaram que neste sistema apenas a Razão de Poisson não influencia de forma significativa o choque entre as partículas e que o coeficiente de restituição só é significativo no termo de interação com o coeficiente de atrito estático. Os demais parâmetros influenciam o valor do ângulo de forma diretamente proporcional. A Simulação 8 foi a que conduziu aos resultados simulados mais próximos do valor experimental do ângulo de repouso estático da soja, que apresentou as seguintes condições. Este tipo de calibração de parâmetros é uma etapa necessária, sendo que os valores desses parâmetros podem ser empregados em simulações de diversos sistemas granulares contendo soja.

\section{AGRADECIMENTOS}

Os autores agradecem o apoio financeiro provido pela FAPEMIG (PCE-00201-14).

\section{REFERÊNCIAS}

CLEARY, P. W. Predicting charge motion, power draw, segregation and wear in ball mills using discrete element methods, Min. Eng., v. 11, p. 1061, 1998.

FAVIER, J.F., ABBASPOUR-FARD, M.H., KREMMER, M., RAJI, A., Shape representation of axi-symmetrical, non-spherical particles in discrete element simulation using multi-element model particles. Eng. Computations, v. 6, p. 467- 480, 1999.

GAO, D., SUN, J. Using DEM in Particulate Flow Simulations. In Hydrodynamics - Optimizing Methods and Tools, pg. 29-50. 1882.

HERTZ, H. On the contact of elastic solids. J. reine und angewandte Mathematik, v. 92, p. 156-171,

MINDLIN, R.D. Compliance of elastic bodies in contact. J. Appl. Mech., v. 16, p. 259-268, 1949.

SANTOS, K. G.; MURATA, V. V.; BARROZO, M. A. S. Three-dimensional computational fluid dynamics modeling of spouted bed. Can. J. Chem. Eng., v. 87, p. 211-219, 2009.

SOUZA, J. Z., Acoplamento entre CFD e DEM para a simulação de escoamentos líquido-sólido na indústria de petróleo. Programa de Pós-Graduação em Engenharia Química, Universidade Federal de Santa Catarina, 2010, 118 p. Dissertação de Mestrado.

TSUJI, Y., TANAKA, T.; ISHIDA, T. Lagrangian numerical simulation of plug flow of cohesionless particles in a horizontal pipe. Powder Technol., v. 71, p. 239-250, 1992.

WALTON, O. R., BRAUN, R. L. Simulation of a rotary drum and repose tests for friction spheres and rigid clusters. Workshop on Flow particulates and fluids, 1993. 